\title{
An IT project management methodology generator based on an agile project management process framework.
}

\author{
Evangelos Markopoulos \\ HULT International Business School, 35 Commercial Road, Whitechapel, E1 1LD London \\ United Kingdom. \\ evangelos.markopoulos@faculty.hult.edu
}

\begin{abstract}
Information Technology Project Management and Software Project Management in particular depends heavily on the project's type and constraints. Quality, financial, technical, schedule, complexity and other constraints affect significantly the management process. Over the last two decades project management methodologies have been developed to support the project management effort. Many methodologies cover generic approaches emphasizing on the planning or estimation activities, others on tracking, others on quality and others on very specific management practices that could support the delivery of very specific projects. This paper introduces an adjustable (agile) project management framework for managing information technology projects of any type. The framework divides the management activities into systems engineering management and systems acquisitions management phases and operates as a methodology generator feed by the project constraints. The project management methodology that derives is a combination of management and engineering phases based on the needs and constraints of each project per case.
\end{abstract}

Keywords: Process $\cdot$ Project $\cdot$ Management $\cdot$ Agile $\cdot$ Engineering $\cdot$ Methodology $\cdot$ Acquisition $\cdot$ System $\cdot$ Software

\section{Introduction}

Managing information technology (IT) has always been a challenge, especially when managing software systems. The failure of many software projects in the 1960s and in the 1970s was the first indication of the upcoming process management difficulties in information technology and the software evolution. Software was delivered late, schedules were unpredictable, projects cost several times the original estimates and often experienced poor performance and quality characteristics [1]. Information technology projects fail at those times due to lack of engineering knowledge and expertise, but also due to the differences that existed between software and hardware engineering against other engineering disciplines [2]. IT and software projects, at those times in particular, were intangible, usually implemented without development standards and management processes, and were mostly considered 'one-off' projects with no repeatability. 
This software crisis [3], [4] has been repeated in the 1980s and continued also in the 1990s and the 2000's as well [5], [6] in all types of organizations involved with software and information technology projects from either the developer / supplier (those who implement technology) perspective or from the customer (those who acquire technology) perspective.

\section{The process adjustability concern.}

The main cause of this software crisis that counts more than 40 years is primarily based on two major factors. First it is the need for adjustability on the management process processes to the project needs for any project, and second is the need for the requirements management process to be taken seriously and followed precisely. [7].

Projects significantly vary on deterministic factors such as size, complexity, budget, time, etc., and nondeterministic factors such as development team maturity, acceptance criteria, process maturity, etc. The adjustability of the management process has a significant, and critical role not only on the technology providers, the ones developing the technology, but also on the customers, the ones acquiring the technology [8], [9]. The proper management process must have the characteristics that will support the efforts and goals of both parties involved in a project.

The management processes that are primarily focused on managing a project with emphasis on its technical challenges, or on its planning or tracking activities, create fuzziness in the interpretation of the implementation and management efforts that need to be placed.

The second factor in the software crisis is clearly focused on the requirements process, a concept that was [10] and will keep on being [11] closely related with the quality and success of a project [12], [13]. The quality of the requirements and the requirements management process is a barometer not only to the success of a project, but also to all planning and management techniques developed around the project. Engineering models, management methodologies, and operations environments are all heavily affected by the quality and the maturity of the project requirements which in turn define the project and impacts its implementation and management strategy.

Having analyzed the prime, and secondary factors of the software and technological crisis, an adjustable and unified project management framework is proposed that can be possibly used effectively and efficiently as a useful tool. However such a framework must have the capability to be scalable to the process and project requirements. This scalability, along with the identities of the framework [14] can be used as a methodology generator, producing adjustable process models, per case and when needed, for all type of projects.

The proposed approach redefines the term 'agility' and kind of renames it to 'adjustability' as the challenge is not only on being flexible, but mostly on being able to continuously adjust to the project needs in order to stay flexible, or agile. 


\section{VR The Adjustable Unified Project Management Framework (AUPMF)}

The Adjustable Unified Project Management Framework (AUPMF) is a concept, which consolidates four project management dimensions into two framework dimensions giving this adjustable project management approach.

The first dimension of the framework is the Systems Engineering Management (SEM) dimension and derives directly from the IT and software engineering project management principles. The engineering framework dimension aims to manage a project from the engineering perspective. This perspective is based on the management of the technical quality, or qualitative management, of the project by managing the development method, the quality of the deliverables produced by the method and other technical documents, validations, verifications and milestones significant to software engineering under the software quality principles.

The second dimension of the framework is the Systems Acquisition Management (SAM) dimension and derives from the consolidation of the Planning, Tracking and Organizational project management approaches. This consolidation aims to manage a project from the pure managerial perspective under the total quality management principles. This is achieved by performing quantitative project management, which is exactly the opposite of the qualitative project management. The quantitative management approach is based on creating estimations and managing those estimations quantitatively by organizing management teams to track what has been planned against what has been done. The qualitative management approach on the other hand, is focused on the management that will achieve technical excellence, not necessarily within time and budget.

Figure 1 describes the formation of the two project management frameworks through the consolidation of the four project management dimensions.

The integration of the two project management frameworks (SEM and SAM) creates an Adjustable Unified Project Management Framework approach, which can cover the needs of almost all types of information technology projects, under all management goals and constraints.

The SEM (Systems Engineering Management) project management framework which is integrated in the AUPMF supports processes that can help the project management efforts and goals from the development point of view. Regardless the nature of the implementation process, managing the engineering process is very crucial to the success of the project or product that is being developed. Also regardless the way the implementation and the software development is executed, either it is in-house development, custom made projects, COTS (Components off the shelve), systems parameterization or systems implementation, the management scope is focused primarily on obtaining technical quality that meets operational expectations, manages constraints and stays within the deadlines. The processes included in the SEM framework are based on the development life-cycle of a system, something that is very critical to be 
followed and managed precisely in order to reach the expected quality which in this case is the prime goal of the SEM approach.

On the other hand, the SAM (Systems Acquisition Management) project management framework which is also integrated in the AUPMF, aims to meet the two other project management key expectations, which are based on the control of budget and the control of the time. Having in the SAM framework all the necessary processes that can provide accurate cost and schedule estimations, the management of a project relies then on the organizational structure, the management team and the tracking model that will verify the control of the estimations and will document the deviations.

The SAM framework approach is primarily used by not-technical project managers or by general project managers by profession, aiming to meet specific deadlines and constrains without much emphasis on the engineering dimension of the project. Meeting time and budget for SAM manages, is more important than meeting the quality of the project.

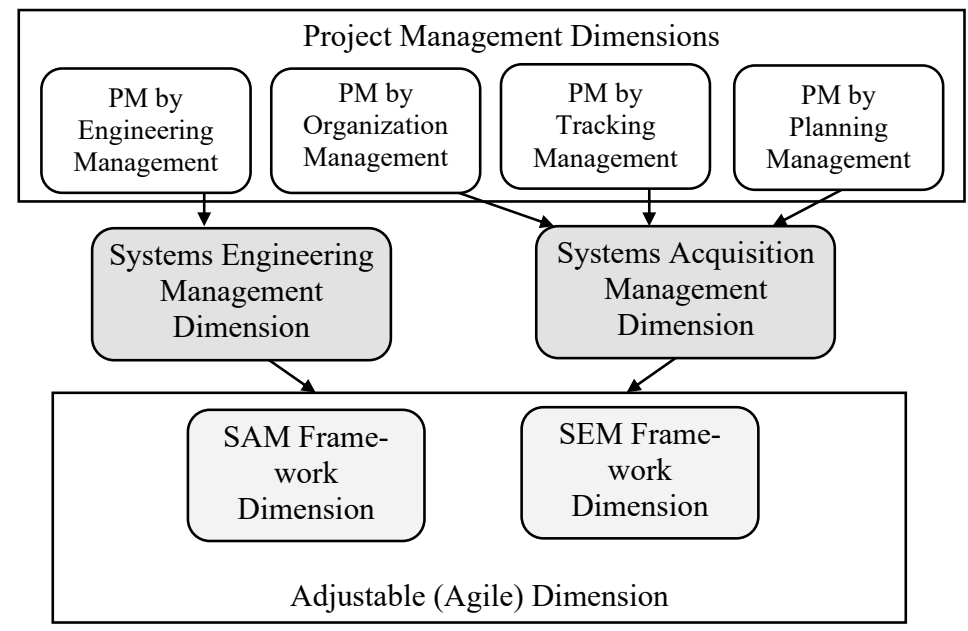

Fig. 1. Project Management Dimension consolidation in the AUPMF

\section{The AUPMF Dimensions Synergy.}

The benefit of the AUPMF and what characterizes it, can be outlined as the synergy among the SAM and the SEM project management dimensions. The framework allows and helps the project manager to select the proper combination of processes from the two management dimensions, and generate a project management approach based on the needs of each project. On the other hand, each project management dimension on AUPMF could also be used as a project management methodology as well, depending on the type of management desired per project implementation. This adjustability and, not agility, is what the AUPMF framework can offer to the mature information 
technology project managers who can think and lead, but not to ones who believe and follow.

This methodology generation process is described in figure 2. By defining the needs of the project to be managed, the phases and process that will be used towards the management of the specific project are selected from the management goals and expectations.

Taking for instance a hardware-oriented project with limited software applications. In this case the project management methodology will be significantly different from the one to be used in a custom-made software business application project. In the first case the management will be much more oriented in the planning and tracking of the project since most of the project components are well developed and tested. In the second case the management of the project will be quality oriented, based on detailed tracking on the engineering practices used to develop the software.

Other project parameters that affect the process structure of the management methodology are the results of an assessment that could take place before project initiation in order to identify the manager's needs and goals, in order for the framework to generate the most appropriate management method.

This methodology parameterization can be repeated for every project. A project manager has actually two sets of processes to work with (SEM and SAM) through which many project management methodologies can be generated if the project requirements and expectations are known, or even unknown in some cases.

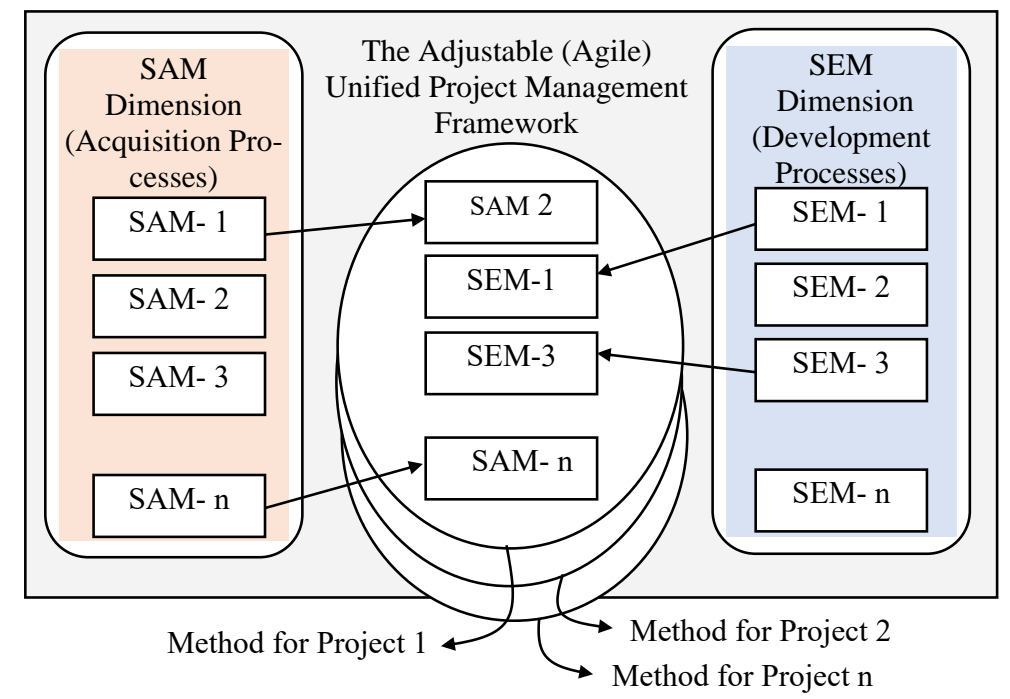

Fig. 2. Project dimension's process synergy 


\section{The AUPMF Process Matrix.}

In order to simplify the complexity of process selection towards the creation and implementation of the desired project management methodology for each project specifically the Adjustable Unified Project Management Framework is based on a requirements interpretation matrix for process generation.

The matrix of AUPMF is a three-tier - two-dimensional matrix. The first tire indicates the available processes from the two process dimensions (SEM and SAM) of the framework. The second tire indicates the selected processes from each process dimension that will form the desired project management method for a specific project. The third tier indicates the relationship among the selected process and their implementation/execution strategy. Figure 3 describes the three AUPMF matrix tiers.

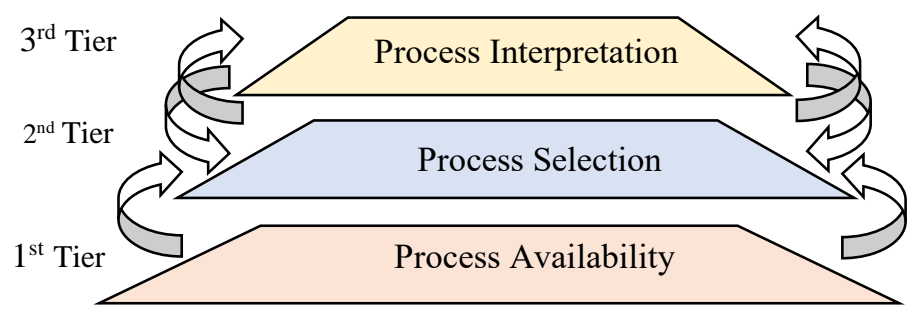

Fig. 3. Three-tier Process Implementation Matrix

The interpretation of the two type of processes in the framework's dimensions form a two-dimensional matrix which is the AUPMF matrix layout as presented in figure 4. The processes of the engineering dimension in the matrix are vertically listed while the processes of the acquisition dimension are horizontally listed.

The way the matrix works is by initially selecting the processes that will be used in the desired project management methodology from each framework dimension (SEM or SAM). Not all SEM or SAM process are selected to be placed in the AUPMF matrix, but only the ones related with the project requirements and management goals.

The selection of the desired processes can be done initially in a conceptual, not precise, manner. By placing each desired process in the matrix, automatically the proposed project management method is generated.

If the project management goal, for example, is to manage the project using the engineering perspective, which is management for the development quality, then the completion of the matrix will start from the engineering processes filling the matrix left to right. Each matrix row indicates the implementation of the specific engineering process on the selected management processes.

If on the other hand the project management goal is to manage the project using the acquisition perspective, which is management by planning and tracking, then the 
completion of the matrix will start from the management processes, filling the matrix top to bottom.

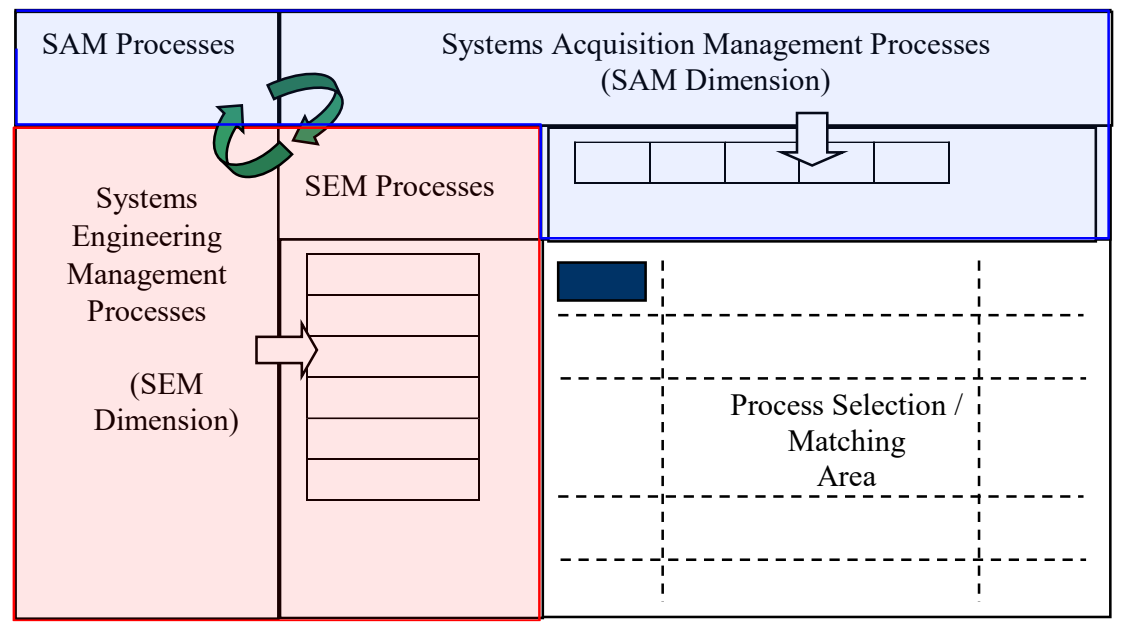

Fig, 4. The AUPMF matrix layout

\section{Reading the AUPMF Matrix}

An example of a semi-complete AUPMF Matrix is shown in figure 5. The interpretation of the project management methodology that derives from this matrix (with the limited indicative values) can be done from the SEM point of view or from the SAM point of view, depending what management approach is to be followed.

\begin{tabular}{|c|c|c|c|c|c|c|c|}
\hline \multicolumn{2}{|c|}{$\begin{array}{l}\text { Application of the } \\
\text { SAM Processes } \\
\text {....on }\end{array}$} & \multicolumn{6}{|c|}{ Systems Acquisition Management Processes } \\
\hline \multirow{7}{*}{$\begin{array}{l}\text { Systems } \\
\text { Engineering } \\
\text { Mgmt } \\
\text { Processes } \\
\text { (SEM } \\
\text { Dimension) }\end{array}$} & $\begin{array}{l}\text { on the SEM } \\
\text { Processes }\end{array}$ & $\begin{array}{c}\text { All } \\
\text { Mgmt } \\
\text { Practices } \\
\end{array}$ & Tracking & $\begin{array}{c}\text { Change } \\
\text { Mgmt }\end{array}$ & $\begin{array}{c}\text { Quality } \\
\text { Assurance }\end{array}$ & .. & $\begin{array}{c}\text { Contract } \\
\text { Mgmt }\end{array}$ \\
\hline & $\begin{array}{l}\text { All Eng. } \\
\text { Practices }\end{array}$ & & 0 & 0 & 1 & 0 & 0 \\
\hline & Req. Mgmt & 0 & 1 & 0 & 0 & & 0 \\
\hline & Syst. Analysis & 0 & 1 & 1 & 0 & & 1 \\
\hline & Coding & 0 & 0 & 1 & 0 & & 0 \\
\hline & $\ldots$ & & & & & & \\
\hline & Testing & 0 & 1 & 0 & 0 & & 1 \\
\hline
\end{tabular}

Fig 5. Example of AUPMF Matrix in Practice 
The interpretation of the matrix from the SEM prospective can be done by reading the matrix rows, Left-to-Right, giving in this case the following project management methodology:

'All project processes in all phases will be subject to quality assurance inspection. In the requirements management process the customer will participate only with tracking activities in the requirements life cycle. In systems analysis, the customer will track the system, and any analysis changes done will be controlled by change management techniques that could affect contract management actions. The coding of the system will be under the programmer's control with no user involvement other that the responsibility to track code changes. The testing of the system will be under the system's tester control with the customer to track the test plan and proceed with contract changes when not satisfied.'

It must be noted that the above methodology derived by interpreting the ' 1 's placed in the matrix. The ' 1 's indicate that the specific cell will be activated, and the output will be the process that will derive by reading the SEM on the SAM process elements.

In a similar way, the interpretation of the matrix from the SAM perspective is based on reading the matrix columns, Top-to-Bottom, giving the following, in this case, project management methodology:

'Quality assurance activities will be applied to all engineering phases. The requirements will be tracked throughout their life cycle for completeness and correctness. Change management activities will be applied on the system analysis and system coding activities that will be delivered by the supplier in order to verify their completeness. Contract management activities will be applied on the systems analysis and systems testing activities in order to verify contractual agreements and possibly modify them if needed'.

The customer and supplier goals differentiate the way the SEM and SAM processes are interpreted. One customer, for example, might need to perform contract management towards tracking the deliverables of the project. if they are specified, in the contract, while another customer might need to perform contract management in order to keep on modifying or updating the contractual obligations of the supplier based on the quality and completeness of the project progress.

\section{Benefits from using the AUPMF.}

The AUPMF can contribute to organizational project management improvement plans in multiple ways. The entire framework is based on the concepts of adjustability and flexibility, in order to help the project's outcome to meet the information systems characteristics, and specifically the software quality characteristics [15].

Any framework, on the other hand, needs to be able to be used in all types of information technology projects, by all type of organizations under all types of technical, financial and management constraints. The structure of the AUPMF allows it to be easily and successfully used by all types of organizations and specifically by the SME's, 
or actually the SISMEs (Software Intensive Small and Medium Size Enterprises) [16]. SISMEs due to their size, time and budget constraints, face practical restrictions on either using specific project management methodologies or creating their own methodology to be used in each project per case.

Project differentiation creates process differentiation and therefore management differentiation. In general, any differentiation creates the need of adjustability, and adjustability requires changes, but changes are hard to be adopted and accepted. Resistance to change is a prime management consideration for applying management and organizational process control models towards process improvement and total quality management [17]. The AUPMF response to this challenge is its user-based adjustability per project and per case.

The framework specifically promotes user involvement since there are no mandating processes, basic models or minimum process requirements in it. The structure of the framework and the flexibility that provides are based totally on its operations model, which is user-driven and purely democratic. The proposed models that derive each time from the framework operations are accepted or enhanced / changed by the participants in the project, and not necessarily by the project managers.

Depending on the way each organization or project manager views the concept of success, the AUPMF has the flexibility to reach and support such success variations.

\section{Risks from using the UPMF.}

Every success factor can be turned into failure factor if not properly interpreted, understood and managed. What can be considered as a benefit can also be considered as a risky, if not properly approached. The freedom provided by the framework through its ability to be adjusted can be very harmful to the ones with no management and process engineering background, knowledge or experience.

Amateur and/or inexperienced project managers can create very complex management methods from using the framework in their attempt to make sure that their project will be well managed monitored and documented. However, the effort to manage such complex methods requires experience in both systems engineering and project management. On the other hand, project managers who might underestimate the complexity of a project can create a project management approach with processes that do not support major management activities and principles.

In order to bypass these risks, extensive training is needed on process improvement, project management and systems engineering only to matured and disciplined personnel.

Management commitment is another risk in the AUPMF. The management needs to support the methods deriving from the AUPMF even if they differ from project to project. After all, this is the major benefit of the framework. All the project participants need to be part of this process when the matrix is being completed; otherwise there are 
no guarantees that the derived management approach will work in practice. The senior management of the organization needs to support these activities regardless if they cost much or take productive time from the participant's busy work schedules.

Finally, the AUPMF is not panacea. The benefits offered require process maturity and management commitment, to work out. Quality is free [18], but only if you do everything right.

\section{$9 \quad$ Results}

Information technology project management and specifically software project management is full of gray areas, unexpected situations, dependencies and ambiguous tradeoffs [19]. Managing information technology projects is very difficult, but it is not impossible. Project management is more about understanding the management needs than the implementation processes, activities and milestones. If you do not know where you are going; no road will help you [20].

Successful project management is based on successful understanding of the project environment and requirements [21]. This diversity on the project management goals puts the project management concept in an endless loop seeking for the silver bullet in a continues evolving industry composed from new process, methods and best practices. Undoubtedly all of the new contributions in the international project management community and discipline are working well, but only under specific conditions and limitations. On the other hand, most of them require significant expertise in order to be followed completely in order to be effective, and others require a bureaucratic mentality to get aligned with their standards [22].

The AUPMF presented in this paper can contribute towards managing software projects and information systems complexity. The framework matrix which is the key element in its operation and interpretation works actually as a methodology generator. A project manager with a defined set of requirements, can create through the framework, the proper process model that can be used towards successfully implementing this specific set of requirements.

The matrix on the other hand, and its process generation capabilities, allows the framework to be easily used for all type of projects regardless their size, volume and complexity. This capability comes to boost up the technocratic development visions of the SMEs and SISMEs, who silently today, support the larger part if the world's economy, but forbidden grow effectively by using proper project management methods and practices do to their size, budget, projects and even culture.

The AUPMF makes process engineering for process management affordable to anyone for anything, at any time. 


\section{References}

1. Brooks F. P.: The Mythical Man Month. Reading MA., Addison Wesley. (1975)

2. Sommerville I.: Software Engineering, 6th Edition, Addison Wesley (2001)

3. Mills E.: Software Metrics, SEI Curriculum Module SEI-CM-12-1.1, (1988)

4. Arthur, L. J. Measuring Programmer Productivity and Software Quality. New York: John Wiley, (1985)

5. Glass R.: Is there really a software crisis, IEEE Software, vol 15, no.1, 104--105 (1998)

6. Gibbs W.: Software's Chronic Crisis, Scientific American, vol. 271, no. 3, 86--95 (1994)

7. Markopoulos E.: Quality Assurance Best Practices for the Implementation of Logistics Information Technology Systems, Proceedings of the 17th International Logistics Congress, Athens Greece, October 2001, vol. 1, 532--557 (200)

8. Hoffmann H. and Geiger J.: Quality Management in Action: a Swiss case study, Vol. 15, No.1, pp35--53 (1995)

9. Herbsleb, J.: Benefits of CMM-based Softtware Process Improvement: Initial Results. Technical Report CMU/SEI-94-TR-13, SEI (1994)

10. Davis G., 'Strategies for Information Requirements Determination', IBM Systems Journal, vol 21., no. 1,4--30 (1982)

11. Wyder T., 'Capturing Requirements with Use-Cases', Software Development, pp 37--40 (1996)

12. Leveson N.: Software Safety: Why, What and How, ACM Computing Surveys, vol. 18, no.2, 125--163, (1986)

13. Robertson S.: Robertson J., Mastering the Requirements Process, Addison Wesley (1999)

14. ISO/IEC 12207:1995: Information Technology-Software life cycle processes - Amendment 1 ', (2002)

15. Dunn R.: Software Quality - Concepts and Plans, Prentice Hall, (1990)

16. Markopoulos E.: An Empirical Adjustable Software Process Assessment Model for Software Intensive Small and Medium Size Enterprises, 7th European Conference on Software Quality, Conference Notes Tammerpaino, Tampere, Finland, 16--19 (2002)

17. Deming W. E.: Out of Crisis, MIT Press, (2000)

18. Crosby P.: Quality Is Free: The Art of Making Quality Certain, McGraw-Hill, (1979)

19. Royce W.: Software Project Management, Addison Wesley, (1998)

20. Humphrey, W. S.: Managing for innovation - Leading Technical People;, Englewood Cliffs, NJ, Prentice Hall, (1987)

21. Reel, J.S.: Critical Success Factors in Software Projects', IEEE Software, May 1999, 106113 (199)

22. Emmerich G.: Managing Standards Compliance, IEEE Transactions on Software Engineering, vol. 25, no. 6. 836--851 (1999) 